\title{
Mortalidade por câncer de próstata no Maranhão no século XXI
}

\author{
Mortality from prostate cancer in Maranhão in the XXI century \\ La mortalidad por cáncer de próstata en Maranhão en el siglo XXI
}

Tarcísio Pires Ribeiro

ORCID: https://orcid.org/0000-0001-6389-0926

Universidade Federal do Maranhão, Brasil

E-mail: tarcisio.ribeiro@discente.ufma.br

Alessandro Santana Padilha

ORCID: https://orcid.org/0000-0003-1573-6937

Universidade Federal do Maranhão, Brasil

E-mail: sandro.ufrn@hotmail.com

Carlos Martins Neto

ORCID: https://orcid.org/0000-0002-6554-3087

Universidade Federal do Maranhão, Brasil

E-mail: carlosneto91@hotmail.com

Aida Patrícia da Fonseca Dias Silva

ORCID: https://orcid.org/0000-0003-0138-8505

Universidade Federal do Maranhão, Brasil

E-mail: aida.patricia@discente.ufma.br

Sâmia Amélia Mendes Silva

ORCID: https://orcid.org/0000-0002-7281-5044

Universidade Federal do Maranhão, Brasil

E-mail: samia_amelia@hotmail.com

Bruno Luciano Carneiro Alves de Oliveira

ORCID: https://orcid.org/0000-0001-8053-7972

Universidade Federal do Maranhão, Brasil

E-mail: oliveira.bruno@ufma.br

\begin{abstract}
Resumo
Introdução: Diferenciais socioeconômicos, raciais e de acesso a medidas de prevenção, diagnóstico e tratamento precoce tem aumentado a mortalidade por câncer de próstata. Objetivo: Descrever a mortalidade por câncer de próstata no Estado do Maranhão, segundo variáveis socioeconômicas e demográficas. Métodos: Estudo descritivo com 4.238 óbitos por câncer de próstata, CID 10 - C61, ocorridos de 2000 a 2017 entre homens $\geq 40$ anos. Foram estimadas as frequências absolutas e relativas. Elaboraram-se curvas etárias de mortalidade proporcional por cor/raça e idade, e box-plot para a idade de morte por cor/raça. Resultados: O câncer de próstata representou 20,0\% dos óbitos por neoplasia. Predominaram entre pardos, $>80$ anos, com baixa escolaridade, com morte domiciliar e na região fora da capital São Luís. Observou-se pior estrutura de mortalidade para pardos e pretos em todos os anos, com a mediana de idade de morte menor do que nos brancos. Conclusões: Observou-se um perfil de características individuais e contextuais associadas aos óbitos, apontando a necessidade de ações mais amplas de prevenção, diagnóstico e tratamento dessa neoplasia.
\end{abstract}

Palavras-chave: Câncer; Mortalidade; Próstata.

\begin{abstract}
Introduction: Socioeconomic and racial differentials and access to prevention, diagnosis and early treatment measures have increased mortality from prostate cancer. Objective: Describe mortality from prostate cancer in the State of Maranhão, according to socioeconomic and demographic variables. Methods: Descriptive study with 4,238 deaths from prostate cancer, CID 10-C61, which occurred from 2000 to 2017 among men $\geq 40$ years old. The absolute and relative frequencies were estimated. Age curves of proportional mortality by color/race and age, and box-plot for the age of death by color/race were drawn up. Results: Prostate cancer accounted for $20.0 \%$ of deaths from cancer. Predominates among browns, $>80$ years old, with low education, with death at home and in the region outside the capital São Luís. There was a worse mortality structure for browns and blacks in all years, with a median age of death lower than in whites. Conclusions: There was a profile of individual and contextual characteristics associated with deaths, indicating the need for actions broader prevention, diagnosis and treatment of this cancer.
\end{abstract}

Keywords: Cancer; Mortality; Prostate. 


\begin{abstract}
Resumen
Introducción: Las diferencias socioeconómicas, raciales y de acceso a las medidas de prevención, diagnóstico y tratamiento precoz han aumentado la mortalidad por cáncer de próstata. Objetivo: Describir la mortalidad por cáncer de próstata en el estado de Maranhão, según variables socioeconómicas y demográficas. Métodos: Estudio descriptivo con 4.238 muertes por cáncer de próstata, CIE 10 - C61, ocurridas entre 2000 y 2017 entre hombres $\geq 40$ años. Se estimaron las frecuencias absolutas y relativas. Se elaboraron curvas de mortalidad proporcional a la edad por color/raza y edad, así como un diagrama de caja para la edad de muerte por color/raza. Resultados: El cáncer de próstata representó el $20,0 \%$ de las muertes por neoplasia. Predominan los morenos, >80 años, con baja escolaridad, con muerte en el hogar y en la región fuera de la capital São Luís. Se observó una mayor estructura de mortalidad para los pardos y los negros en todos los años, con una mediana de edad de muerte menor que la de los blancos. Conclusiones: Se observó un perfil de características individuales y contextuales asociadas a las muertes, lo que apunta a la necesidad de acciones más amplias de prevención, diagnóstico y tratamiento de esta neoplasia.
\end{abstract}

Palabras clave: Cáncer; Mortalidade; Próstata.

\title{
1. Introdução
}

Em todo o mundo, o câncer de próstata é um dos tumores com maior incidência e mortalidade. Representa, a quinta causa de morte por neoplasia e a segunda entre os homens. A mortalidade por esse agravo tem estacionado em níveis elevados nas últimas décadas (Brasil, 2021; Rawla, 2019). Em 2018, foram estimados mais de 300.000 óbitos no mundo, sendo a região da América Latina a que teria apresentado a $2^{a}$ maior taxa de mortalidade, atrás apenas do Caribe e Sudeste da África (Rawla, 2019; Merriel et al., 2018). O Brasil, seria o país com a $2^{\mathrm{a}}$ maior taxa de morte daquela região, sendo que são estimados cerca de 65.840 novos casos e 15.391 óbitos por ano no país (Brasil, 2021; Tourinho et al., 2016).

Um conjunto de fatores de risco tem sido bem estabelecidos como associados ao câncer de próstata, entre eles a idade, a cor/raça negra (preta e parda), comportamentos de saúde (tabagismo, obesidade, inatividade física, dietas e vida sexual), histórico familiar e genético, e as localidades menos desenvolvidas, que apresentam maior privação socioeconômica e de acesso e uso de serviços de saúde do país (Braga et al., 2017; Braga; De Souza, 2017; Ferlay et al., 2015). O estado do Maranhão configura-se entre os estados mais pobres da federação e o onde os comportamentos negativos à saúde e o menor acesso aos serviços de saúde ainda são um dos grandes problemas a saúde pública, principalmente entre homens (IBGE, 2021). Em 2019, entre os estados do Nordeste, o Maranhão esteve entre os três maiores em número de novos casos, atrás apenas dos estados do Ceará e Bahia, sendo o câncer de próstata a principal causa de morte neoplásica no estado (Brasil, 2021).

Logo, diferenciais socioeconômicos, raciais e de acesso a medidas de prevenção, diagnóstico e tratamento precoce tem gerando excesso de mortalidade por câncer de próstata nas diferentes localidades do Brasil. Contudo, analises desse tipo com dados do Maranhão ainda não foram realizadas. Nesse contexto, torna-se importante conhecer a ocorrência da doença a partir da análise de informações dos óbitos, pois estes podem revelar o padrão de mortalidade ao longo dos anos no estado, suas diferenças individuais e contextuais, ao mesmo tempo contribuir com o debate sobre a melhoraria e ampliação aos cuidados a saúde do homem.

Assim, este estudo buscou descrever a mortalidade por câncer de próstata no Estado do Maranhão, segundo variáveis socioeconômicas e demográficas.

\section{Metodologia}

Trata-se de estudo descritivo baseado nos dados secundários de mortalidade por câncer de próstata como causa básica de óbito. Foram utilizados dados de morte por câncer de próstata com CID 10: C61. Os dados foram obtidos por meio do Sistema de Informação de Mortalidade (SIM) do Departamento de Informática do Sistema Único de Saúde (DATASUS).

Foram analisados 4.238 óbitos ocorridos no estado do Maranhão entre 2000 a 2017 e restritos a homens $\geq 40$ anos de idade, por ser considerada a idade em que ações clínicas de controle desse agravo devem ser iniciadas. 
A pesquisa foi desenvolvida no Estado do Maranhão que possui uma população estimada em 2019 em 7.075.181 habitantes, área de 332.936,948 km, densidade demográfica 19,81 hab/km com total de 217 municípios sendo São Luís a capital do estado (IBGE, 2021). No ano de 2019 a população masculina correspondia a 3.261,515 habitantes, sendo 814.154 entre 40 a 90 anos (IBGE, 2021; Brasil, 2018). Este estado tem uma conformação 08 (oito) Macrorregiões: Balsas, Caxias, Coroatá, Imperatriz, Pinheiro, Presidente Dutra, Santa Inês e São Luís. Considerando a necessidade do Estado de ter um Sistema de Saúde com resolutividade segundo a Comissão Intergestores Bipartite - CIB/MA Resolução: CIB/MA No 44/2011, de 16 de junho de 2011.

Foram estimadas as frequências absolutas e relativas para os óbitos segundo um conjunto de variáveis socioeconômicas e demográficas: sexo, cor/raça, faixa etária em anos, escolaridade em anos, estado civil, local de ocorrência e o município e regional de residência do óbito. Para ambos os sexos, as idades foram consideradas em cinco grupos etários (40-49, 50-59, 60$69,70-79, \mathrm{e} \geq 80$ anos de idade). Foram elaboradas também curvas etárias de mortalidade proporcional por cor/raça para os cinco grupos de idade estudados segundo período agregado de três anos (2000 a 2002, 2003 a 2005, 2006 a 2008, 2009 a 2011, 2012 a 2014, 2015 a 2017). Por fim, para esses mesmos períodos de anos, elaboraram-se box-plot para a idade de morte por cor/raça.

Todas as etapas realizadas até a geração do banco de dados, utilizou-se o software Excel, em seguida, os dados foram exportados para análise pelo software estatístico Stata 14.0. Os dados foram então apresentados em gráficos e tabelas.

A pesquisa foi elaborada com agregados de dados secundários disponíveis on-line, os quais não contêm informações sigilosas que permitam a identificação dos sujeitos. Assim, a foi dispensada a sua aprovação por Comitê de Ética em Pesquisa. O estudo foi realizado em conformidade com a Resolução n 510, de 7/04/2016, do Conselho Nacional de Saúde.

\section{Resultados}

Entre 2000 e 2017, no estado do Maranhão ocorreram 194.117 óbitos entre homens $\geq 40$ anos, sendo $10,9 \%$ deles por neoplasias. O câncer de próstata foi responsável por 20,0\% desses óbitos ( $\mathrm{n}=4.238$ casos) no estado nesse período. A figura 1 apresentou variação proporcional desses óbitos entre 2000 e 2017. No período aumentou 41,7\% a frequência dos óbitos. O pico inicial de aumento foi entre o ano 2000 e 2006, quanto a partir desse ano se estabilizou, passando a representar cerca de $1 / 4$ de todos os óbitos por neoplasias entre homens $\geq 40$ anos. (Figura 1 ). 
Figura 1: Proporção de óbitos por câncer de próstata entre os óbitos por neoplasias entre homens $\geq 40$ anos no Maranhão entre os anos 2000 e 2017.

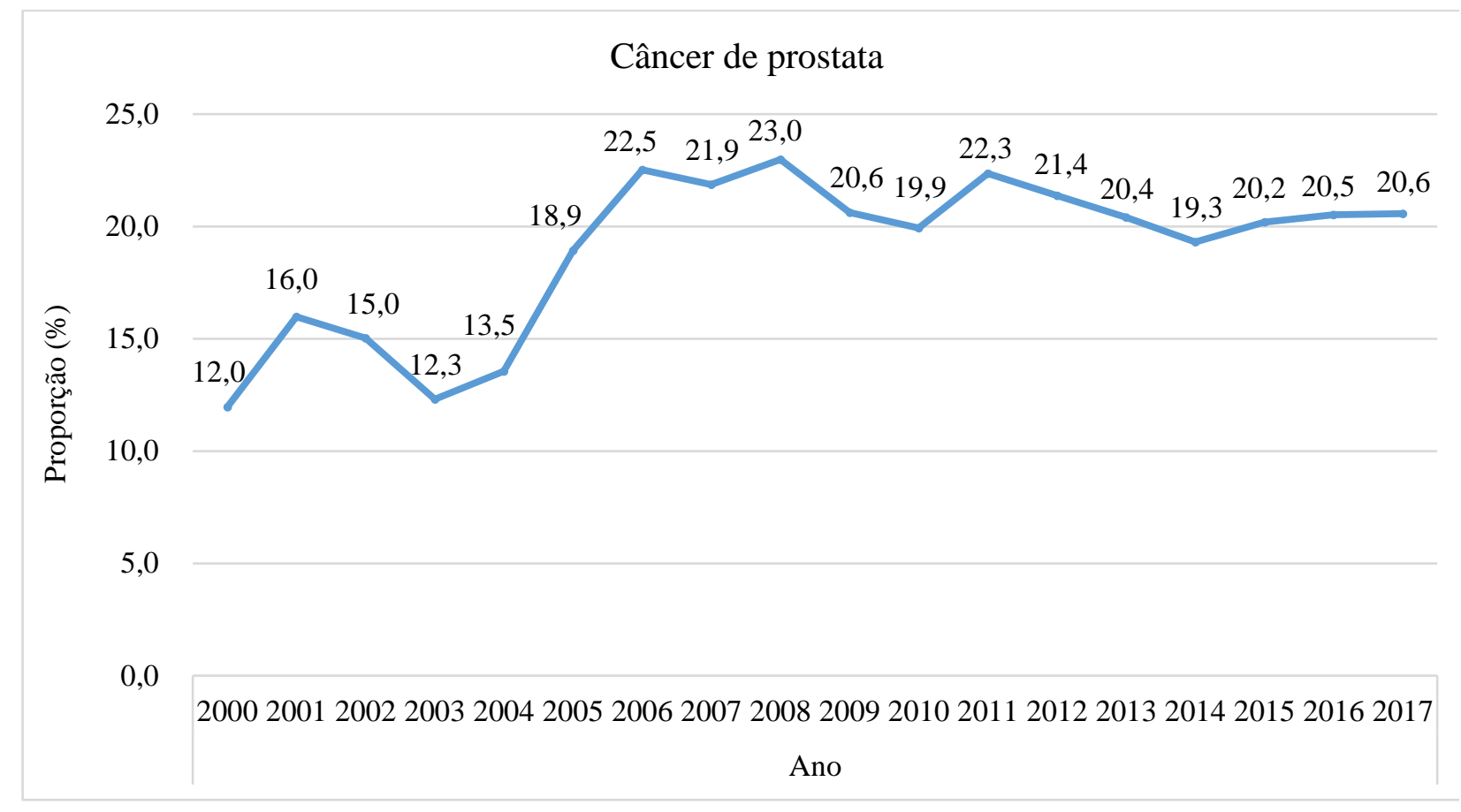

Fonte: Dados disponíveis no Datasus (Brasil, 2019).

Predominaram óbitos por câncer de próstata nos pardos (61,0\%). A prevalência desses óbitos aumentou com a idade, sendo mais frequente nas faixas etárias de 70 a $79 \operatorname{anos}(35,4 \%)$ e $\geq 80$ anos $(41,5 \%)$. Homens com ensino fundamental incompleto e sem nenhuma escolaridade, somados apresentam 74,8\% dos óbitos, e foi menos frequente óbitos no maior nível de escolaridade (3,3\%). A maioria dos óbitos foram entre os casados (64,3\%) e ocorreu nos domicílios (54,6\%), seguido do ambiente hospitalar (44,1\%). Todas as Macrorregiões de Saúde do estado foram acometidas por câncer de próstata, sendo a maior proporção nas Regiões de Saúde da cidade de São Luís (38,4\%), seguido de Imperatriz (13,1\%) e Presidente Dutra (11,9\%). (Tabela 1). 
Tabela 1: Distribuição absoluta e relativa (\%) de óbitos por câncer de próstata ( $\mathrm{N}=4.228)$ segundo as características sociodemográficas no Maranhão de 2000 a 2017.

\begin{tabular}{|c|c|c|}
\hline \multirow{2}{*}{ Variáveis } & \multicolumn{2}{|c|}{ Óbitos por câncer de próstata } \\
\hline & $\mathbf{n}$ & $\%$ \\
\hline \multicolumn{3}{|l|}{ Cor/raça } \\
\hline Brancos & 1.014 & 25,0 \\
\hline Pardos & 2.471 & 61,0 \\
\hline Pretos & 566 & 14,0 \\
\hline \multicolumn{3}{|l|}{ Faixa etária (em anos) } \\
\hline 40 a 49 & 32 & 0,8 \\
\hline 50 a 59 & 211 & 5,0 \\
\hline 60 a 69 & 733 & 17,3 \\
\hline 70 a 79 & 1.499 & 35,4 \\
\hline$\geq 80$ & 1.753 & 41,5 \\
\hline \multicolumn{3}{|l|}{ Escolaridade $\left(\mathrm{em}\right.$ anos) ${ }^{2}$} \\
\hline Nenhuma & 1.791 & 47,5 \\
\hline 1 a 3 & 1.029 & 27,3 \\
\hline 4 a 7 & 578 & 15,3 \\
\hline 8 a 11 & 248 & 6,6 \\
\hline$\geq 12$ & 126 & 3,3 \\
\hline \multicolumn{3}{|l|}{ Estado Civil $^{3}$} \\
\hline Solteiro & 681 & 17,9 \\
\hline Casado & 2.445 & 64,3 \\
\hline Viúvo & 610 & 16,1 \\
\hline Separado & 64 & 1,7 \\
\hline \multicolumn{3}{|l|}{ Local de ocorrência ${ }^{4}$} \\
\hline Hospital/outro serviço de saúde & 1.861 & 44,1 \\
\hline Domicílio & 2.306 & 54,6 \\
\hline Via pública & 17 & 0,4 \\
\hline Outros & 39 & 0,9 \\
\hline \multicolumn{3}{|l|}{ Macrorregião do estado Maranhão } \\
\hline São Luís & 1.625 & 38,4 \\
\hline Caxias & 375 & 8,9 \\
\hline Pinheiro & 272 & 6,4 \\
\hline Imperatriz & 554 & 13,1 \\
\hline Presidente Dutra & 502 & 11,9 \\
\hline Coroatá & 454 & 10,7 \\
\hline Santa Inês & 295 & 7,0 \\
\hline Balsas & 151 & 3,6 \\
\hline
\end{tabular}

Notas: Foram considerados nas estimativas das variáveis os dados sem a informação: 1 - Cor/raça (amarelos=19, indígenas= 10, ignorados=148) 2- Escolaridade $(n=456)$; 3- estado civil $(n=428)$; 4- Local de ocorrência $(n=5)$.

Fonte: Dados disponíveis no Datasus (Brasil, 2019).

As curvas etárias de mortalidade proporcional por cor/raça segundo o período agregado de três anos revelaram padrão temporal semelhante de distribuição dos óbitos. Ao longo dos anos, permaneceu sem alterações a pior estrutura relativa de mortalidade para pardos e pretos em relação aos brancos. As diferenças foram apenas na magnitude dessas proporções ao longo dos anos. Entre brancos, predominou a mortalidade na maior faixa etária $\geq 80$ anos em todos os períodos de anos avaliados, e essa proporção aumentou com o tempo. Já para pardos e pretos os óbitos predominaram na faixa etária anterior entre 70 e 79 anos, e verificou-se gradiente racial desfavorável aos pretos em relação aos pardos. Ao longo dos anos, observou-se discreta evolução no ganho de anos de vida representada pelo aumento da proporção de óbitos entre idosos com $\geq 80$ anos de todos os grupos raciais, porém esse ganho foi maior para brancos, permanecendo a estrutura de desvantagens para pardos e pretos. (Figura 2). 
Verificou-se diferenças importantes na mediana da idade de morte entre os grupos raciais segundo os períodos de anos avaliados. Em geral, pardos e pretos sempre apresentaram mediana de idade de morte menor que os brancos. Entre os anos de estudo, houve até redução das diferenças entre as medianas de idade com aumento na idade de morte para pardos e pretos, porém nestes permaneceram sempre menor estimativa que nos brancos (Figura 3).

Figura 2: Curvas de mortalidade proporcional por cor/raça segundo período agregado de anos no Maranhão entre 2000 e 2017.

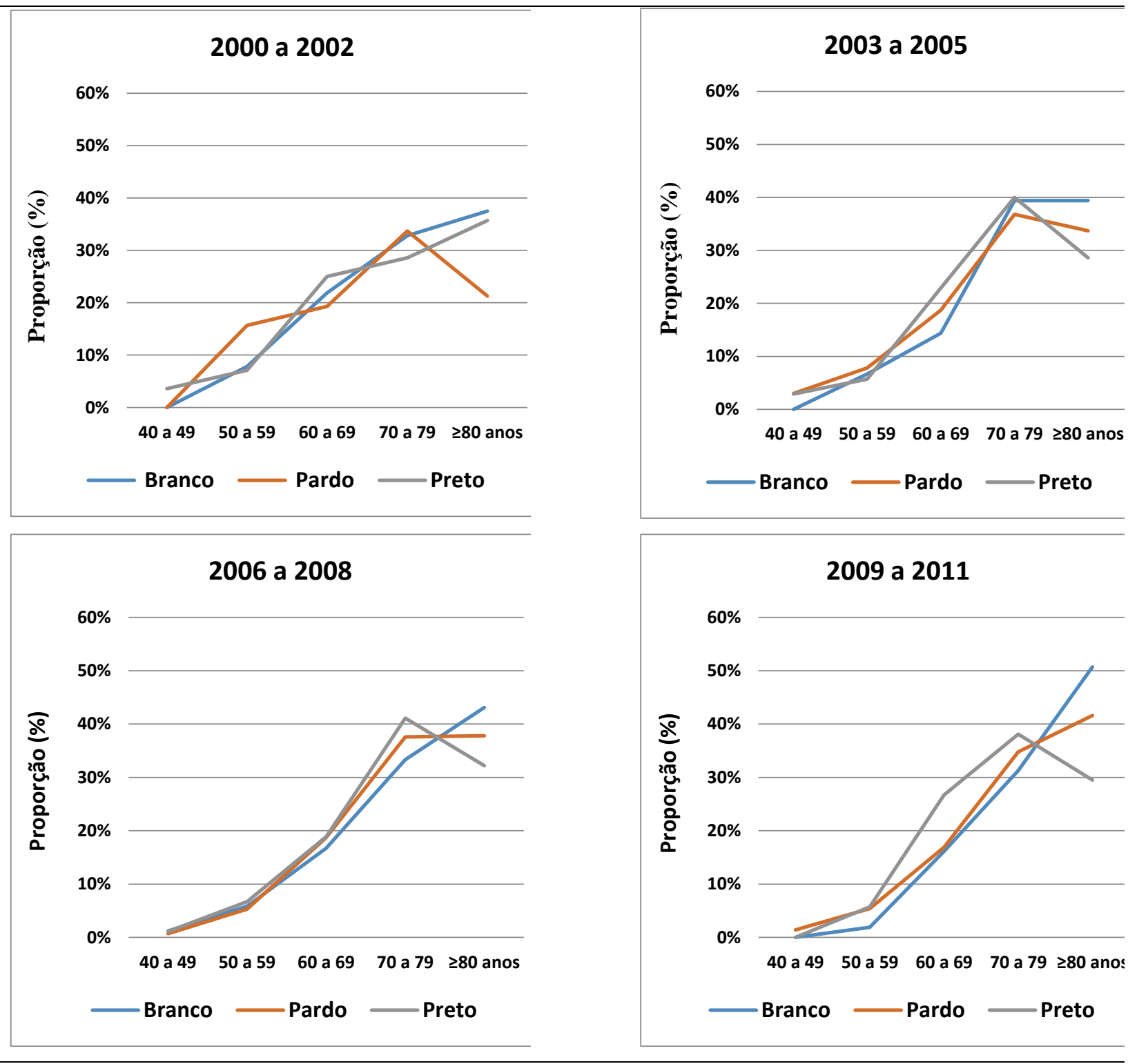


Research, Society and Development, v. 10, n. 8, e48810817621, 2021

(CC BY 4.0) | ISSN 2525-3409 | DOI: http://dx.doi.org/10.33448/rsd-v10i8.17621

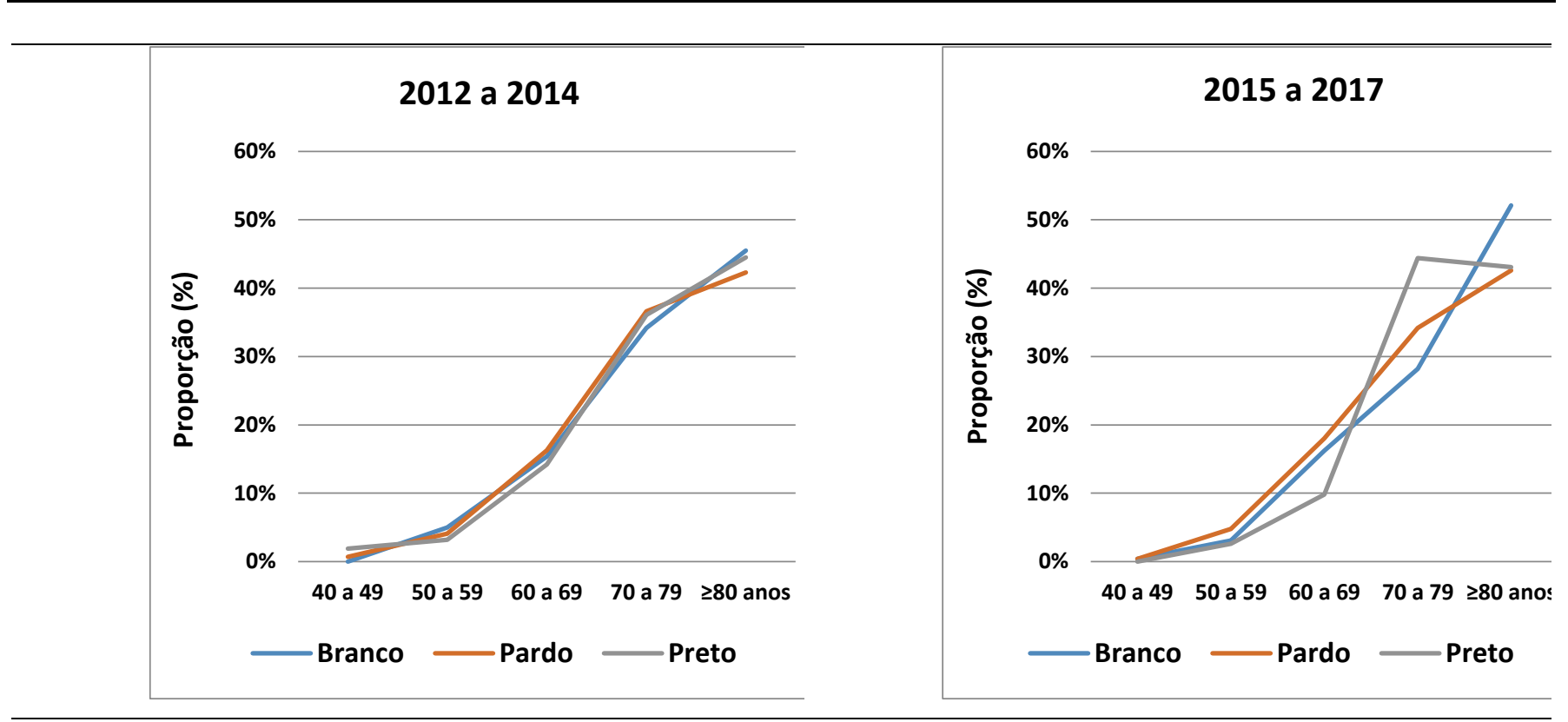

Fonte: Dados disponíveis no Datasus (Brasil, 2019). 
Figura 3: Box-plot das idades de morte por câncer de próstata por cor/raça segundo período agregado de anos no Maranhão entre 2000 e 2017.
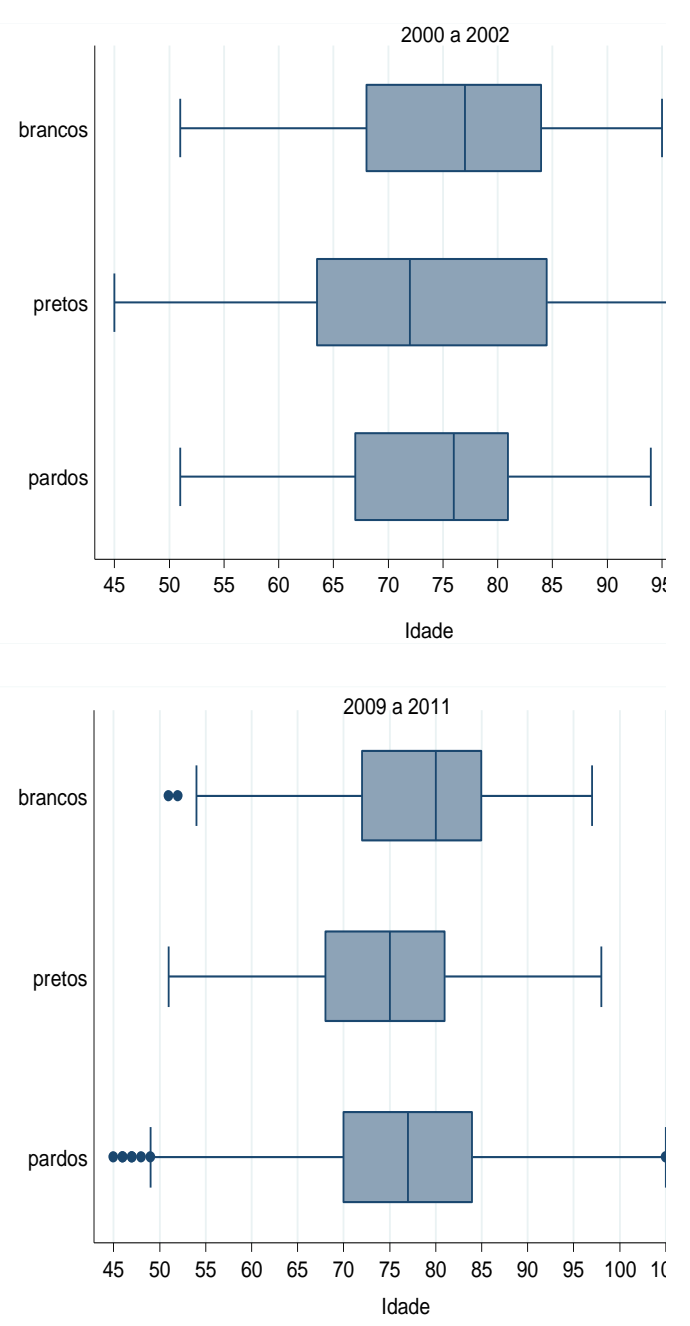
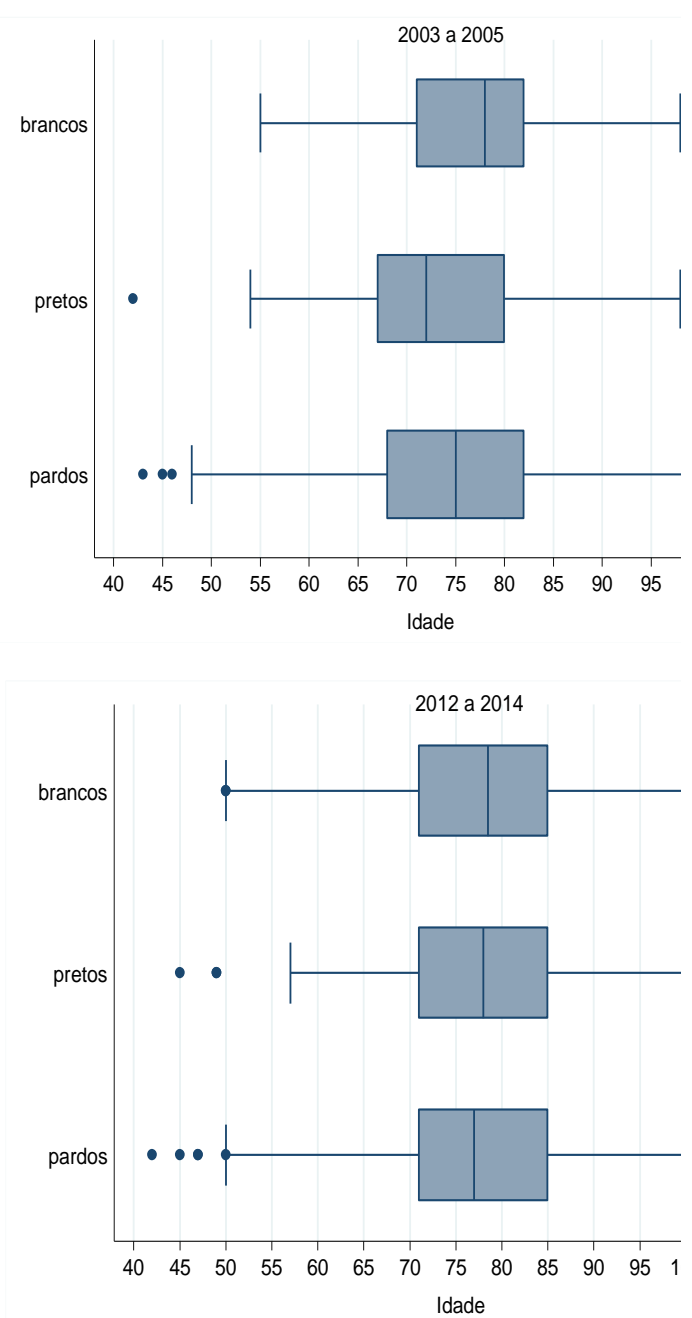

2006 a 2008

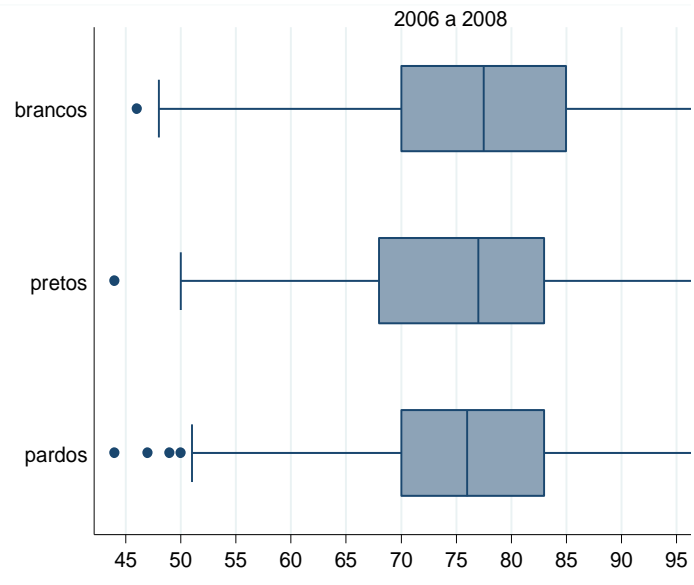

dade

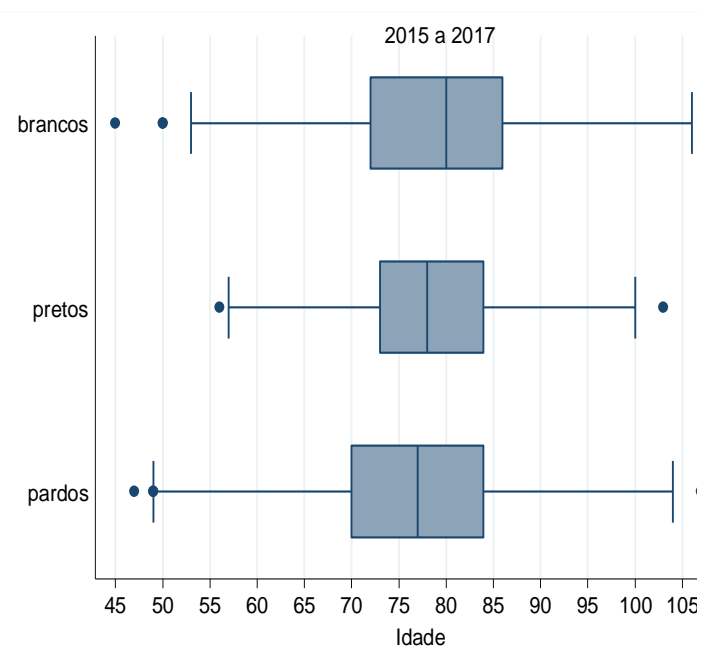

Fonte: Dados disponíveis no Datasus (Brasil, 2019). 
Research, Society and Development, v. 10, n. 8, e48810817621, 2021

(CC BY 4.0) | ISSN 2525-3409 | DOI: http://dx.doi.org/10.33448/rsd-v10i8.17621

\section{Discussão}

Os resultados deste estudo indicaram grandes desigualdades na composição da mortalidade por câncer de próstata no Maranhão. Foi elevada proporção de óbitos por câncer de próstata entre todos os óbitos por neoplasia de homens no estado, representando cerca de $1 / 4$ de todos os óbitos. Observou-se um perfil de características associadas a maior ocorrência desses óbitos, representadas por maior proporção entre pardos, de baixa escolaridade, que evoluíam para estágio terminal da doença com óbito domiciliar e distribuídos em todas as regiões de saúde do estado, mas com a maioria ocorrendo fora da região da capital São Luís.

As piores curvas de mortalidade proporcional por cor/raça apontaram mortes mais precoces para pardos e pretos do que para brancos. Nestes, a morte foi mais frequente na faixa de idade mais elevada. As desigualdades observadas refletem um conjunto de desvantagens estruturais e aspectos da organização social e dos serviços de saúde no Maranhão. Nossos resultados apontam que a raça influencia as trajetórias de vida dos indivíduos e gera desfavorável risco de morte para pardos e pretos ao longo dos ciclos de vida e gerações.

Esses achados se assemelham aos encontrados em outros estudos, que destacaram o aumento do número de óbitos por câncer de próstata em homens acima de 40 anos em outros estados do Brasil (Araújo et al., 2019; Migowski, 2010; Nascimento et al., 2011; Souza Junior, 2019; Zacchi et al., 2019). Porém, a prevalência de mortes por este agravo no estado foi maior do que a observada em para todo o país e outros países (Merriel et al., 2018; Tourinho et al., 2016; Enemugwem et al., 2019).

Tende-se atribuir essa realidade a situação aos aspectos socioeconômicos e geográficos específicos do estado, que interferem no acesso aos serviços especializados e à ampliação do tempo entre diagnóstico e tratamento (Braga, 2021). As dificuldades de acesso à saúde e a precariedade da rede assistencial de saúde, podem estar contribuindo para o comportamento da mortalidade, observado nesta pesquisa, pois tais casos tendem a ser mais identificados em estágios e idades avançados, quando não há mais possibilidade de tratamento (Silva, 2020). Estima-se que 1/3 dos casos de neoplasia da próstata possa ser prevenido e outro terço possa ser curado se detectado precocemente em programas de rastreio (Enemugwem et al., 2019).

Revisão sistemática encontrou um padrão consistente de diferenças por desvantagem residencial em todo o continuum do câncer de próstata, desde o teste de PSA até a incidência, estadiamento, tratamento, sobrevivência e mortalidade. Especificamente, em comparação com residentes de áreas afluentes, homens que vivem em áreas socioeconomicamente desfavorecidas em geral tiveram menor teste de PSA e incidência de câncer de próstata, mais disseminação avançada da doença no diagnóstico, menor sobrevida e maior mortalidade (Dasgupta et al., 2019).

A presente pesquisa permitiu verificar que homens mais velhos apresentaram maiores prevalências de óbitos por câncer de próstata. Estudos evidenciaram (Araújo et al., 2019; Junior, 2019; Silva, 2020; Panis et al., 2018) essa informação também, mostrando que homens mais jovens são diagnosticados em estádios mais iniciais da doença e apresentam maior sobrevida quando comparado a homens com idade acima dos 50 anos (Bernard et al., 2020; Lima et al., 2018). A idade avançada é fator de risco conhecido na história da evolução do câncer de próstata. Considerada como doença da terceira idade no homem, apresenta crescimento lento do tumor (Panis et al., 2018). Com isso é provável que o aumento de sua incidência, em parte deva-se ao aumento da expectativa de vida e rotinas do sistema de saúde (Zacchi et al., 2019). Estudos estimam aumento da incidência global de câncer de próstata com o aumento da expectativa de vida (Rawla, 2019; Tourinho et al., 2016; Teoh et al., 2019).

O baixo nível de escolaridade está associado aos maiores casos de óbitos nesse estudo, sendo um achado comum em analises de perfil de óbitos por câncer de próstata (Viana et al., 2014; Zacchi et al., 2019). É fundamental destacar que a baixa participação em programas de rastreamento é um fenômeno multifatorial, mas que tende a interagir com a escolaridade (Sá et al., 2020; Batista et al., 2013).

Verificou-se maiores casos de óbitos em homens casados quando comparadas aos homens viúvos e solteiros, esse 
Research, Society and Development, v. 10, n. 8, e48810817621, 2021

(CC BY 4.0) | ISSN 2525-3409 | DOI: http://dx.doi.org/10.33448/rsd-v10i8.17621

achado corrobora com os resultados de outros estudos (Araújo et al., 2019; Nascimento et al., 2011). Esse comportamento pode estar relacionado ao papel desempenhado pelo vinculo família. Onde a família contribui positivamente com os hábitos de saúde do homem, auxiliando em momentos de agravamento e estabilidade clínica. Com isso homens casados tem mais acesso aos serviços de saúde onde os casos de câncer são notificados (Nascimento et al., 2011; Lima et al., 2018). Ao contrário do que encontramos quando observamos homens viúvos e solteiros. Estes em sua maioria negligenciam sua saúde, fazendo com que possíveis casos de neoplasia da próstata não sejam conhecidos pelo sistema de saúde (Rauber, 2018).

Pode-se perceber que os maiores números de óbitos por local de ocorrência, foram em ambiente domiciliar. Isso pode estar relacionado ao quadro de evolução do câncer, onde pacientes com diagnósticos e estados terminais, são orientados a proceder com tratamentos paliativos em suas casas (Atty, 2018; SBU, 2021). O câncer é uma das doenças que mais demandam cuidado paliativo no atendimento domiciliar, e o de próstata é que mais gera atendimento nos programas domiciliares em homens (Atty, 2018; Vasconcelos et al., 2018).

No presente estudo, homens pretos/pardos apresentaram maior risco de mortalidade quando comparados aos de cor branca. Esses dados são semelhantes aos de outros autores (Araújo et al., 2019; Zacchi et al., 2019). Pretos e pardos possuem os piores indicadores de escolaridade, estão inseridos nos piores postos de trabalho e têm menos acesso à saúde, bens e serviços sociais (Batista et al., 2013). Somada as variáveis socioeconômicas em conjunto com crenças e atitudes culturais que poderiam em grande parte explicar o efeito observado (Lannin et al., 1998).

Tendência de redução da mortalidade por câncer de próstata por tem sido observada nos EUA em todos os grupos raciais, mas tem permanecido desigualdades desfavoráveis aos negros e hispânicos (Rawla, 2019). Entre negros, a questão da idade de morte e quanto ela é mais prematura relação aos brancos, sugere a estrutura de desigualdades mais desfavoráveis aos negros que tendem morrem em maior prevalência e ainda mais jovens do que os brancos. Para alguns autores, essas desvantagens nos indicadores de mortalidade podem refletir a interação de características genéticas com as socioeconômicas. Entre negros a característica racial seria associada ao maior risco de câncer de próstata e a formas mais agressivas, o que destaca que a falta de adequado rastreio e o atraso no tratamento repercutem mais negativamente esse grupo (Rawla, 2019). Com isso, diferenciais raciais no estágio de detecção e a qualidade do tratamento, associados a distribuição dos riscos socioeconômicos e de saúde no estado do Maranhão podem explicar as desigualdades raciais na característica etária das mortes por câncer de próstata verificadas neste estudo.

Existem algumas limitações neste estudo. Possíveis subnotificações dos casos de óbitos ou mesmo a incompletude de variáveis nas DO ainda é um problema sobretudo em óbitos fora do ambiente hospitalar e até mesmo aos óbitos por câncer de próstata em áreas remotas de difícil acesso ao sistema de saúde. Contudo, a qualidade dos dados vem melhorando nos últimos e análises de agregados de series de dados tendem a sofrer menos com essa limitação. Outra questão é a classificação raça/cor da pele. Nas declarações de óbito espera-se que a declaração do quesito racial seja atribuída por um familiar, porém é possível que o profissional médico responsável utilize diferentes critérios de classificação e tende de classificar racialmente as pessoas levando em conta o nível socioeconômico, seja por suas visões de mundo, juízo de valor ou mesmo por pressão social (Junior, 2019).

Assim, é possível que as desigualdades raciais aqui observadas possam estar distorcidas, porém a estrutura de mortalidade racial ao longo dos anos avaliados sugere que esses diferenciais seriam sistemáticos e parecem ter profundas raízes e associação com a precária realidade econômica e acesso a serviços sociais e de saúde que pardos e pretos têm em relação aos brancos. Assim, essas limitações não apagam a relevância das iniquidades aqui observadas.

\section{Conclusão}

O câncer de próstata é uma doença que se espera que se torne mais prevalente em uma população que envelhece e sem correção das desigualdades estruturais. Análise da mortalidade no Maranhão por esse agravo revelou um perfil de características 
individuais e contextuais associadas aos óbitos, o que pode indicar a complexa interação de processos sociais, raciais, genéticos, ambientais e comportamentais que existem no Maranhão. Logo, esses resultados apontaram significativas desigualdades e implicações para alocação de recursos dentro dos sistemas de saúde no intuito de se garantir amplas ações de prevenção, diagnóstico e tratamento dessa neoplasia em todos os grupos raciais, especialmente aqueles mais vulnerabilizados como os pardos e pretos.

\section{Referências}

Araújo, M. S. M., Sardinha, A. H. de L., Neto, J. A. de F., Silva, E. L. da, \& Lopes, M. L. H. (2019). Caracterização sociodemográfica e clínica de homens com câncer de próstata. Revista de Salud Pública, 21(3), 1-6. https://doi.org/10.15446/rsap.V21n3.70678

Atty, A. T. de M., \& Tomazelli, J. G. (2018). Cuidados paliativos na atenção domiciliar para pacientes oncológicos no Brasil. Saúde em Debate, 42, 225-236. https://doi.org/10.1590/0103-1104201811618

Batista, L. E., Monteiro, R. B., \& Medeiros, R. A. (2013). Iniquidades raciais e saúde: O ciclo da política de saúde da população negra. Saúde em Debate, 37, $681-690$

Bernard, B., Burnett, C., Sweeney, C. J., Rider, J. R., \& Sridhar, S. S. (2020). Impact of age at diagnosis of de novo metastatic prostate cancer on survival. Cancer, 126(5), 986-993. https://doi.org/10.1002/cncr.32630

Braga, S. F. M., de Souza, M. C., \& Cherchiglia, M. L. (2017). Time trends for prostate cancer mortality in Brazil and its geographic regions: An age-periodcohort analysis. Cancer Epidemiology, 50, 53-59. https://doi.org/10.1016/j.canep.2017.07.016

Braga, S. F. M., Silva, R. P. da, Guerra, A. A., \& Cherchiglia, M. L. (2021). Prostate Cancer Survival and Mortality according to a 13-year retrospective cohort study in Brazil: Competing-Risk Analysis. Revista Brasileira de Epidemiologia, 24. https://doi.org/10.1590/1980-549720210006

Braga, S. F. M., Souza, M. C. de, Oliveira, R. R. de, Andrade, E. I. G., Acurcio, F. de A., \& Cherchiglia, M. L. (2017). Patient survival and risk of death after prostate cancer treatment in the Brazilian Unified Health System. Revista de Saúde Pública, 51, 46-46. https://doi.org/10.1590/s1518-8787.2017051006766

Brasil (2021). Estimativa 2020: incidência de câncer no Brasil / Instituto Nacional de Câncer José Alencar Gomes da Silva.

Dasgupta, P., Baade, P. D., Aitken, J. F., Ralph, N., Chambers, S. K., \& Dunn, J. (2019). Geographical Variations in Prostate Cancer Outcomes: A Systematic Review of International Evidence. Frontiers in Oncology, 9, 238. https://doi.org/10.3389/fonc.2019.00238

Enemugwem, R. A., Eze, B. A., Ejike, U., Asuquo, E. O., \& Tobin, A. (2019). Prostate cancer screening: Assessment of knowledge and willingness to screen among men in Obio Akpor LGA, Rivers State, Nigeria. African Journal of Urology, 25(1), 11. https://doi.org/10.1186/s12301-019-0010-5

Ferlay, J., Soerjomataram, I., Dikshit, R., Eser, S., Mathers, C., Rebelo, M., Parkin, D. M., Forman, D., \& Bray, F. (2015). Cancer incidence and mortality worldwide: Sources, methods and major patterns in GLOBOCAN 2012. International Journal of Cancer, 136(5), E359-386. https://doi.org/10.1002/ijc.29210

Instituto Brasileiro de Geografia e Estatística - IBGE (2021). Cidades: 2020. https://cidades.ibge.gov.br/brasil/ma/panorama.

Brasil (2018). Banco de dados do Sistema Único de Saúde-DATASUS 2018. www2.datasus.gov.br.

Júnior, E. V. de S., Santos, M. S. dos, Nunes, C. A., Souza, Á. R., Trindade, L. E. S., Maia, T. F., Barros, V. S., \& Cruz, D. P. (2019). Perfil de morbiletalidade e impacto econômico por neoplasia maligna prostática. Revista de Enfermagem UFPE on line, 13. https://doi.org/10.5205/1981-8963.2019.240679

Lannin, D. R., Mathews, H. F., Mitchell, J., Swanson, M. S., Swanson, F. H., \& Edwards, M. S. (1998). Influence of socioeconomic and cultural factors on racial differences in late-stage presentation of breast cancer. JAMA, 279(22), 1801-1807. https://doi.org/10.1001/jama.279.22.1801

Lima, A. P. de, Lini, E. V., Giacomazzi, R. B., Dellani, M. P., Portella, M. R., \& Doring, M. (2018). Prevalência e fatores associados à realização de exames de câncer de próstata em idosos: Estudo de base populacional. Revista Brasileira de Geriatria e Gerontologia, 21, 53-59. https://doi.org/10.1590/198122562018021.170054

Merriel, S. W. D., Funston, G., \& Hamilton, W. (2018). Prostate Cancer in Primary Care. Advances in Therapy, 35(9), 1285-1294. https://doi.org/10.1007/s12325-018-0766-1

Migowski, A., \& Silva, G. A. e. (2010). Sobrevida e fatores prognósticos de pacientes com câncer de próstata clinicamente localizado. Revista de Saúde Pública, 44, 344-352. https://doi.org/10.1590/S0034-89102010000200016

Nascimento, A. R. A. do, Trindade, Z. A., Gianordoli-Nascimento, I. F., Pereira, F. B., Silva, S. A. T. da C., \& Cerello, A. C. (2011). Masculinidades e práticas de saúde na região metropolitana de Belo Horizonte-MG. Saúde e Sociedade, 20, 182-194. https://doi.org/10.1590/S0104-12902011000100020

Panis, C., Kawasaki, A. C. B., Pascotto, C. R., Justina, E. Y. D., Vicentini, G. E., Lucio, L. C., \& Prates, R. T. C. (2018). Critical review of cancer mortality using hospital records and potential years of life lost. Einstein (São Paulo), 16(1). https://doi.org/10.1590/S1679-45082018AO4018

Rawla, P. (2019). Epidemiology of Prostate Cancer. World Journal of Oncology, 10(2), 63-89. https://doi.org/10.14740/wjon1191

Rauber, B. R., Rocha, J. de P., Neves, B. B., Seibel, R., Bós Â. G. (2018). Características sociodemográficas de estilo de vida e de acesso a serviços de saúde entre realizadores ou não de exame de próstata. Temas em Saúde, 18(2):124-44. 
Research, Society and Development, v. 10, n. 8, e48810817621, 2021

(CC BY 4.0) | ISSN 2525-3409 | DOI: http://dx.doi.org/10.33448/rsd-v10i8.17621

Sá, R. L. de, Rodrigues, Y. A., Oliveira, E. H. de, \& Britto, M. H. M. (2020). Mortalidade por neoplasia maligna do colo do útero no estado do Maranhão: Perfil epidemiológico e tendência. Research, Society and Development, 9(4), e13942876-e13942876. https://doi.org/10.33448/rsd-v9i4.2876

Silva, D. A. da. (2020). Neoplasia maligna da próstata no Brasil: Morbidade (2013-2018) e mortalidade (2008-2017). Research, Society and Development, 9(6), e178963657-e178963657. https://doi.org/10.33448/rsd-v9i6.3657

Sociedade Brasileira de Urologia (2021). Câncer de Próstata - SBU - SP. http://sbu-sp.org.br/publico/cancer-de-prostata-3/

Teoh, J. Y. C., Hirai, H. W., Ho, J. M. W., Chan, F. C. H., Tsoi, K. K. F., \& Ng, C. F. (2019). Global incidence of prostate cancer in developing and developed countries with changing age structures. PloS One, 14(10), e0221775. https://doi.org/10.1371/journal.pone.0221775

Tourinho-Barbosa, R. R., Pompeo, A. C. L., \& Glina, S. (2016). Prostate cancer in Brazil and Latin America: Epidemiology and screening. International Braz j Urol, 42, 1081-1090. https://doi.org/10.1590/S1677-5538.IBJU.2015.0690

Vasconcelos, G. B., \& Pereira, P. M. (2018). Cuidados paliativos em atenção domiciliar: Uma revisão bibliográfica. Revista de Administração em Saúde, 18(70), Article 70. https://doi.org/10.23973/ras.70.85

Viana, M., Martins, J. T., Maciel, A. A., Marcon, S. S., \& Ribeiro, R. P. (2014). Perfil epidemiológico do homem com câncer de próstata atendido em um hospital universitário. Cogitare Enfermagem, 19(2), Article 2. https://doi.org/10.5380/ce.v19i2.31540

Zacchi, S. R., Viana, K. C. G., Brandão-Souza, C., Amorim, M. H. C., \& Zandonade, E. (2019). Mortalidade em Homens com Câncer de Próstata e sua Associação com Variáveis Sociodemográficas e Clínicas. Revista de Pesquisa Cuidado é Fundamental Online, 11(3), 648-654. https://doi.org/10.9789/21755361.2019.v11i3.648-654 